\title{
An Anti-Ramsey Condition on Trees
}

\author{
Michael E. Picollelli \\ Department of Mathematical Sciences, \\ Carnegie Mellon University, \\ Pittsburgh, PA 15213
}

Submitted: Feb 23, 2007; Accepted: Jan 25, 2008; Published: Feb 4, 2008

Mathematics Subject Classifications: 05C05, 05C15, 05C55

\begin{abstract}
Let $H$ be a finite tree. We consider trees $T$ such that if the edges of $T$ are colored so that no color occurs more than $b$ times, then $T$ has a subgraph isomorphic to $H$ in which no color is repeated. We will show that if $H$ falls into a few classes of trees, including those of diameter at most 4 , then the minimum value of $e(T)$ is provided by a known construction, supporting a conjecture of Bohman, Frieze, Pikhurko and Smyth.
\end{abstract}

\section{Introduction}

Let $\mathbb{P}$ denote the set of positive integers. Let $H=(V, E)$ be a graph, $b \in \mathbb{P}$, and $c$ be a coloring of the edges of $H$, i.e. $c: E \rightarrow X$ where $X$ is a set of colors. We say that $c$ is $b$-bounded if $\left|c^{-1}(x)\right| \leq b$ for all $x \in X$. We say a subgraph $U$ of $H$ is rainbow with respect to $c$ if $c$ is injective on the edges of $U$. Unlike traditional Ramsey theory, which focuses on questions regarding monochromatic copies of $H$ in colorings of a larger graph $G$, anti-Ramsey theory focuses on questions regarding rainbow copies of $H$. For example, Erdős, Simonovits, and Sós considered the minimum number of colors $x$ required so that every coloring of $K_{n}$ using exactly $x$ colors produces a rainbow $H$ [2]. Lefmann, Rödl and Wysocka considered the same problem but with restricted colorings, including $b$-bounded colorings [3]. Bohman, Frieze, Pikhurko and Smyth considered the probabilistic issue of the threshold for the random graph $G_{n, p}$ to asymptotically almost surely contain a rainbow $H$ under any $b$-bounded coloring [1]. In doing so, they explored the following question: if $H$ is a tree, what is the minimum size of a tree $T$ that yields a rainbow $H$ under every $b$-bounded coloring?

Notation 1. Let $H$ and $T$ be trees. We say that $T \rightsquigarrow(H ; b)$ if every b-bounded coloring of the edges of $T$ induces a rainbow copy of $H$ in $T$. Let

$$
A R(H ; b):=\min \{e(T): T \rightsquigarrow(H ; b)\},
$$


where $e(T)$ is the number of edges in $T$.

We henceforth assume that $H$ is a finite tree. The first natural question is whether a finite $T$ exists such that $T \rightsquigarrow(H ; b)$ for every choice of $H$, i.e. if $A R(H ; b)<\infty$. The second natural question is whether or not we can determine $A R(H ; b)$. The first question is answered in [1] by construction, which yields a partial answer to the second.

Definition 1. For a tree $H=(V, E)$, and any two edges e, $f \in E$, let $d(e, f)$ denote the distance between the vertices corresponding to $e$ and $f$ in $\mathcal{L}(H)$, the line graph of $H$. Let $b \in \mathbb{P}$, and let

$$
F(H, e ; b)=\sum_{f \in E} b^{d(e, f)}
$$

and

$$
G(H ; b)=\min _{e \in E} F(H, e ; b) .
$$

In [1], the authors show that

$$
A R(H ; b) \leq G(H ; b)
$$

by constructing a tree $B_{H, e, b}$ for each $e \in E(H)$, called the $b$-blow-up of $H$ centered at $e$, such that $e\left(B_{H, e, b}\right)=F(H, e ; b)$ and $B_{H, e, b} \rightsquigarrow(H ; b)$. (The proof of $(1)$, including the definition of $B_{H, e, b}$, is contained at the end of this section.) Furthermore, they conjecture that this bound is sharp for all trees:

Conjecture 1 (Bohman, Frieze, Pikhurko, Smyth [1]). For all trees $H, A R(H ; b)=$ $G(H ; b)$.

Bohman, Frieze, Pikhurko and Smyth verified Conjecture 1 for paths, rooted trees with a constant branching factor (i.e. all leaves are at the same depth, and all non-leaves have the same degree) and for trees constructed by adding leaves to one end of a 3-path.

In this paper, we approach the problem in the following inductive way. Given $H, H^{\prime}$ will be a carefully chosen subtree formed by removing some of the leaves of $H$. Given any tree $T$ such that $T \rightsquigarrow(H ; b)$, we construct a subtree $T^{\prime}$ of $T$ such that $T^{\prime} \rightsquigarrow\left(H^{\prime} ; b\right)$ and $e(T)-e\left(T^{\prime}\right) \geq G(H ; b)-G\left(H^{\prime} ; b\right)$. If Conjecture 1 holds for $H^{\prime}$, then it holds for $H$. Our first application of this method will be to trees of diameter at most 4 :

Theorem 1. Let $H$ be a tree of diameter at most 4 . Then $A R(H ; b)=G(H ; b)$.

With additional structure on $H$ and $H^{\prime}$, we can use this method to prove a stronger result. Suppose $A R\left(H^{\prime} ; b\right)=G\left(H^{\prime} ; b\right)$, and for any tree $U$ with $U \rightsquigarrow\left(H^{\prime} ; b\right), U$ has at least as many leaves as the minimum-size $b$-blow up of $H^{\prime}$. If $H$ can be constructed from $H^{\prime}$ by adding a constant number of leaves to each leaf of $H^{\prime}$, then $A R(H ; b)=G(H ; b)$ and for all trees $T$ with $T \rightsquigarrow(H ; b), T$ has at least as many leaves as the minimum-size $b$-blow-up of $H$ contains. Thus, proceeding by induction, we can construct a large class of trees for which Conjecture 1 holds. To formalize this idea, we introduce the following definitions. 
Definition 2. Let $H$ be a tree, let $e \in E(H)$, and let $L_{H}=\{f \in E(H): \exists u \in$ $f$ with $d(u)=1\}$ be the set of "edge" leaves of $E(H)$. Then we define

$$
L(H, e ; b):=\sum_{f \in L_{H}} b^{d(e, f)} .
$$

We note that $L(H, e ; b)$ is the number of edge leaves in the b-blow-up $B_{H, e, b}$.

Definition 3. For $b \in \mathbb{P}$, let $\mathcal{S}_{b}$ denote the set of trees $H$ such that $A R(H ; b)=G(H ; b)$ and, for any tree $T$ such that $T \rightsquigarrow(H ; b), T$ has at least $L(H, e ; b)$ leaves, where $e \in E(H)$ satisfies $F(H, e ; b)=G(H ; b)$. Let

$$
\mathcal{S}=\bigcap_{b \in \mathbb{P}} \mathcal{S}_{b}
$$

Definition 4. Let $H$ be a tree. For $k \in \mathbb{P}$, let $H(k)$ be the tree constructed by adding $k$ leaves to every leaf of $H$. For $k_{1}, \ldots, k_{n} \in \mathbb{P}$, inductively define $H\left(k_{1}, \ldots, k_{n}\right)=$ $H\left(k_{1}, \ldots, k_{n-1}\right)\left(k_{n}\right)$.

Theorem 2. Let $H$ be a tree with $e(H) \geq 2$, and let $b, k \in \mathbb{P}$. If $H \in \mathcal{S}_{b}$, then $H(k) \in \mathcal{S}_{b}$.

Theorem 2 provides us with a method to construct trees in $\mathcal{S}_{b}$ from trees known to lie in it, but it does not provide us with examples of trees that actually lie in any $\mathcal{S}_{b}$, let alone $\mathcal{S}$. To remedy this, we show that if $H$ is a path, star, or of diameter $3, H\left(k_{1}, \ldots, k_{n}\right) \in \mathcal{S}$ for all $k_{1}, \ldots, k_{n} \in \mathbb{P}$, provided $H\left(k_{1}, \ldots, k_{n}\right)$ is not a path of length 2 .

Notation 2. For $n \geq 0$, we let $P_{n}$ denote a path on $n+1$ vertices, and $S_{n}$ denote a star graph on $n+1$ vertices.

Corollary 1. If $H$ is $P_{n}$ for $n \neq 2, S_{n}$ for $n \geq 3, P_{2}(k)$ for $k \geq 2$ or of diameter 3 , then $H \in \mathcal{S}$.

The methods we employ in this paper, however, have serious limitations. The first is that they require that an edge $e \in E\left(H^{\prime}\right)$ which minimizes $F\left(H^{\prime}, e ; b\right)$ also minimizes $F(H, e ; b)$. We know, however, that this is not always the case: consider the tree $U$ formed by taking a 3-path and adding one leaf to one end, and $m$ leaves to the other end. If we let $e$ be the edge centered on the original 3-path, and $f$ be the edge adjacent to $e$ and incident with the vertex of degree $m+1$, we have

$$
F(U, e ; b)=(m+1) b^{2}+2 b+1, \text { and } F(U, f ; b)=b^{3}+b^{2}+(m+1) b+1 .
$$

If $b<m-1, G(H ; b)=F(H, f ; b)$, while if $b \geq m-1, G(H ; b)=F(H, e ; b)$. This example also illustrates a second limitation: the edge $e$ that minimizes $F(H, e ; b)$ can depend on $b$, while our techniques so far have only analyzed the structure of $H$ independent of $b$. One way to avoid this peril is to take the asymptotic route: by fixing $H$ and letting $b \rightarrow \infty$, the conjecture suggests the choice of $e$ should lie centermost on a longest-path. We will show that this is precisely the case for trees formed by adding leaves to the ends of a path. 
Theorem 3. Let $k \geq h \in \mathbb{P}$, and let $H$ be the tree constructed by connecting the central vertex of an $S_{h}$ to the central vertex of an $S_{k}$ by a path of length $n \in \mathbb{P}$. Then, provided $b \geq\left(\frac{h+1}{h}\right)(k-h)+1$,

$$
A R(H ; b)=G(H ; b)= \begin{cases}(h+k) b^{r}+\sum_{i=1}^{r-1}\left(2 b^{i}\right)+1 & \text { if } n=2 r-1, \\ h b^{r+1}+k b^{r}+b^{r}+\sum_{i=1}^{r-1}\left(2 b^{i}\right)+1 & \text { if } n=2 r .\end{cases}
$$

Another limitation to this approach is the idea that the structure of $H$ necessarily induces structure on all $T$ such that $T \rightsquigarrow(H ; b)$. Taking $H$ to be a path of length 2 , we have $G(H ; b)=b+1$ but any tree with $b+1$ edges necessarily contains a rainbow copy of $H$. We mention that this will require care in our proof of Theorem 1.

The remainder of the paper is organized as follows: proofs that paths, stars, and trees of diameter 3 lie in $\mathcal{S}$, as well as some necessary structural results, will be covered in Section 2. The proof of Theorem 1 will follow in Section 3. Proofs of Theorem 2 and Corollary 1 lie in Section 4. Finally, the proof of Theorem 3 will follow in Section 5.

Proof of (1). (Bohman, Frieze, Pikhurko, Smyth [1]) Let $e=\{x, y\}$ be an edge of $H$.

Definition 5. We define the b-blow-up of $H$ centered at e, $B_{H, e, b}$, as follows: for each $v \in V(H)$, let $l_{v}=\min \{d(v, x), d(v, y)\}$, and let $S_{v}$ be the set of strings of the form $\left(v, i_{1}, i_{2}, \ldots, i_{l_{v}}\right)$, where $i_{j} \in\{1,2, \ldots, b\}$.

Define the vertex set of $B_{H, e, b}$ to be $\bigcup_{v \in V(H)} S_{v}$. Define the edge set of $B_{H, e, b}$ as the pair $\{(x),(y)\}$ and all pairs $\left\{\left(v, i_{1}, \ldots, i_{l_{v}}\right),\left(w, j_{1}, \ldots, j_{l_{w}}\right)\right\}$, where $\{w, v\} \in E(H), l_{w}=l_{v}+1$, and $i_{k}=j_{k}$ for $k=1, \ldots, l_{v}$.

The $b$-blow-up can be viewed as an algorithmic construction as follows: treat $H$ as a rooted tree with the edge $e$ as its root (rather than a vertex). Starting with $i=0$, for each vertex $u$ of depth $i$ ( $x$ and $y$ have depth 0 in this construction) replace each of $u$ 's downward branches with $b$ copies. Increment $i$ and repeat until the depth of $H$ is reached. One can easily show that these definitions are equivalent, and that $B_{H, e, b}$ is a tree.
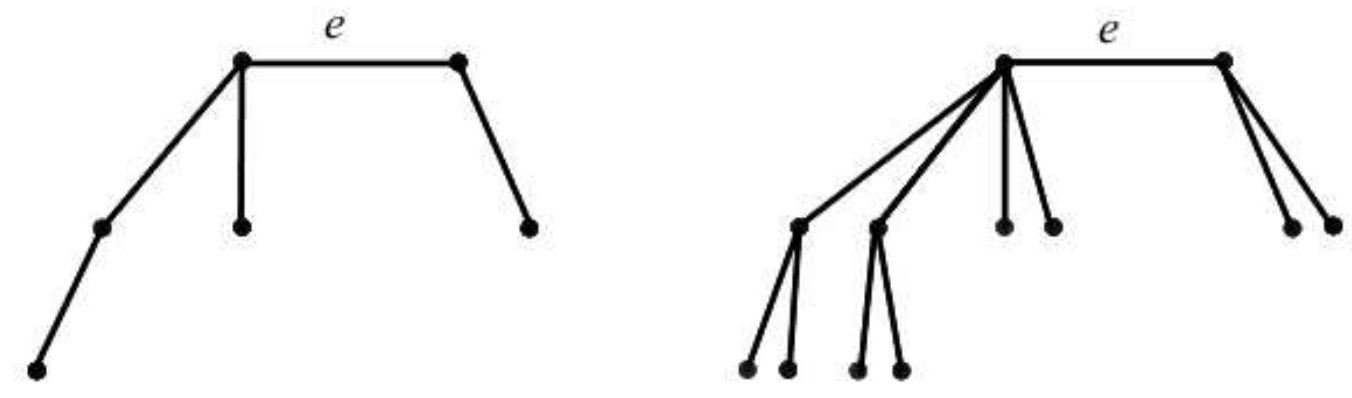

Figure 1: A tree $H$ with edge $e$ and the 2-blow-up $B_{H, e, 2}$.

Our interest lies in showing that $B_{H, e, b} \rightsquigarrow(H ; b)$ and $e\left(B_{H, e, b}\right)=F(H, e ; b)$, which together imply (1).

We call the set of edges between a vertex in $S_{v}$ and all adjacent vertices in $S_{w}$, where $l_{w}=l_{v}+1$, a bundle, as well as the singleton containing $\{(x),(y)\}$, so that the set $\mathcal{B}$ of 
bundles partitions the edge set of $B_{H, e, b}$. Let $c$ be a $b$-bounded coloring of $B_{H, e, b}$, and for each $B \in \mathcal{B}$, let $C_{B}$ be the set of colors used on edges in $B$. Then, for any $Y \subseteq \mathcal{B}$, we have

$$
\left|\bigcup_{B \in Y} C_{B}\right| \geq \frac{1}{b} \sum_{B \in Y}|B| \geq \frac{(|Y|-1) b+1}{b}
$$

and hence $\left|\bigcup_{B \in Y} C_{B}\right| \geq|Y|$. By Hall's Theorem, there exists a system of distinct representatives of the sets of bundles, call it $Z$.

As $Z$ contains an edge from every bundle, the subgraph of $B_{H, e, b}$ containing those edges contains a copy of $H$. Since each edge in $Z$ has a different color, that copy is rainbow. Therefore, $B_{H, e, b} \rightsquigarrow(H ; b)$.

For $v \in V(H) \backslash\{x, y\}$, letting $f_{v}$ be the unique edge incident with $v$ on the path from $v$ to, say, $x$ in $H, l_{v}$ is precisely $d\left(e, f_{v}\right)$, the distance from $e$ to $f_{v}$ in $\mathcal{L}(H)$. Therefore

$$
e\left(B_{H, e, b}\right)=\left(\sum_{v \in V(H)}\left|S_{v}\right|\right)-1=1+\sum_{v \in V(H) \backslash\{x, y\}} b^{l_{v}}=1+\sum_{f \in E(H) \backslash\{e\}} b^{d(e, f)}=F(H, e ; b) .
$$

\section{Preliminary Results}

Clearly $A R\left(P_{1} ; b\right)=1=G\left(P_{1} ; b\right)$ and $A R\left(P_{2} ; b\right)=b+1=G\left(P_{2} ; b\right)$, so our focus will be on larger trees. By convention, we will often refer to edges of a tree incident with a vertex of degree 1 as "leaves". Additionally, as we are only considering $b$-bounded colorings, we will simply refer to them as "colorings" (or "partial colorings").

Prior to establishing the theorems, we need several preliminary results. In Section 2.1 we will show that if $e(H) \geq 3$ and $H$ is a path (Lemma 1 and Corollary 2), a star (Lemma 2 ), or of diameter 3 (Lemma 3 ) then $H \in \mathcal{S}$.

Then, in Section 2.2, we will generalize our methods in a way more suitable for the applications that follow. The colorings we use will be constructed locally to force restrictions on where particular subtrees of any rainbow $H$ in $T$ can lie. To that end, we will introduce the notion of clumped vertices and that of forbidding sets of vertices. The former allows us to keep track of the vertices and edges "outside" of a subtree $U$ of $T$ in a very natural way. The latter notion will allow us to guide our selection of a subtree $T^{\prime}$ by restricting which vertices in a rainbow $H$ in $T$ can lie outside $T^{\prime}$ under suitable partial colorings.

\subsection{Paths, Stars, and Trees of Diameter 3}

Notation 3. Let $T$ be a tree and $U$ be a subtree of $T$. For every $v \in V(T)$, let $N(v)=$ $N_{T}(v)=\{w \in V(T): v w \in E(T)\}$ denote the neighborhood of $v$ and $d(v)=d_{T}(v)=$ $|N(v)|$ the degree of $v$. Similarly, for $u \in V(U)$, let $N_{U}(u)=N_{T}(u) \cap V(U)$ and $d_{U}(u)=$ $\left|N_{U}(v)\right|$. 
Let $\Delta(T)=\max \{d(v): v \in V(T)\}$ be the maximum degree of $T$, and let $L(T)=\{v \in$ $V(T): d(v)=1\}$ denote the set of (vertex) leaves of $T$.

If $T$ is a rooted tree with root $v$, for every $u \in V(T)$, let $\hat{N}(u)=\{w \in V(T)$ : $w$ is a child of $u\}$ and $\hat{d}(u)=|\hat{N}(u)|$.

Lemma 1. If $n \geq 3$ and $T \rightsquigarrow\left(P_{n} ; b\right), T$ contains at least $A R\left(P_{n-2} ; b\right)+1$ vertices of degree at least $b+1$.

The proof of Lemma 1 will follow Lemma 5 in Section 2.2.

\section{Corollary 2.}

$$
\begin{gathered}
A R\left(P_{2 r-1} ; b\right)=1+\sum_{i=1}^{r-1} 2 b^{i}=G\left(P_{2 r-1} ; b\right), \text { and } \\
A R\left(P_{2 r} ; b\right)=1+\sum_{i=1}^{r-1} 2 b^{i}+b^{r}=G\left(P_{2 r} ; b\right) .
\end{gathered}
$$

Additionally, if $n \geq 3$ then $P_{n} \in \mathcal{S}$.

Proof. By our comments earlier, we know Corollary 2 holds for $P_{1}$ and $P_{2}$, so suppose $n \geq 2$ (and hence $r \geq 2$ ). Here we consider the odd case; the even case is analogous.

Letting $e$ be the edge centermost on $P_{2 r-1}$ gives $F\left(P_{2 r-1}, e ; b\right)=1+\sum_{i=1}^{r-1} 2 b^{i}$ and $L(H, e ; b)=2 b^{r-1}$ by direct computation. We will show that these provide the appropriate lower bounds by induction on $r$.

Let $T \rightsquigarrow\left(P_{2 r-1} ; b\right)$, and let $B=\{v \in V(T): d(v) \geq b+1\}$. By Lemma 1 and induction, $|B| \geq A R\left(P_{2 r-3} ; b\right)+1 \geq\left(1+\sum_{i=1}^{r-2} 2 b^{i}\right)+1$. We now use the fact that if $\emptyset \neq X \subseteq V(T) \backslash L(T)$, then $|L(T)| \geq \sum_{x \in X} d(x)-2(|X|-1)$, which can easily be shown by induction on $|X|$. Then, since $d(v) \geq b+1>1$ for all $v \in B$,

$$
\begin{aligned}
|L(T)| & \geq \sum_{v \in B} d(v)-2(|B|-1) \\
& \geq(b+1)|B|-2(|B|-1) \\
& =(b-1)|B|+2 \\
& \geq(b-1)\left(1+\sum_{i=1}^{r-2} 2 b^{i}+1\right)+2 \\
& =2 b^{r-1} .
\end{aligned}
$$

Noting that $e(T) \geq|B|+|L(T)|-1$ completes the proof.

Lemma 2. $S_{n} \in \mathcal{S}$ for $n \geq 3$.

Proof. Let $T \rightsquigarrow\left(S_{n} ; b\right)$. Taking any edge $e$ of $S_{n}, F\left(S_{n}, e ; b\right)=L\left(S_{n}, e ; b\right)=(n-1) b+1$, so it suffices to show $|L(T)| \geq(n-1) b+1$. 
Let $v \in V(T)$ with $d(v)=\Delta(T)$. Root $T$ at $v$. Then for every $u \in V(T)$, color the edges between $u$ and $\hat{N}(u)$ using $\lceil\hat{d}(u) / b\rceil$ colors. Let $w$ be the center vertex of a rainbow $S_{n}$ in $T$. If $w=v$, then $\hat{d}(v) / b>(n-1)$, and hence $|L(T)| \geq d(v)=\hat{d}(v) \geq(n-1) b+1$.

Otherwise, $w$ has $1+\lceil\hat{d}(w) / b\rceil \geq n$ colors on incident edges (by including the edge connecting it to its parent), and therefore $\lceil\hat{d}(w) / b\rceil \geq n-1$, so $\hat{d}(w) \geq(n-2) b+1$ and $d(w) \geq(n-2) b+2$. Since $d(v) \geq d(w) \geq 2,|L(T)| \geq 2((n-2) b+2)-2=2(n-2) b+2$ by our comment in the proof of Corollary 2 , and $2(n-2) b+2 \geq(n-1) b+1$ as $n \geq 3$.

Lemma 3. If $H$ is a tree of diameter 3 , then $H \in \mathcal{S}$.

Proof. Showing $A R(H ; b)=G(H ; b)$ can be done directly by choosing the interior edge $e$ of $H$ : then $(e(H)-1) b+1 \leq A R(H ; b) \leq F(H, e ; b)=(e(H)-1) b+1$. Additionally, $L(H, e ; b)=(e(H)-1) b$. Let $T \rightsquigarrow(H ; b)$, and let $L=L(T)$. The remainder of this proof will be devoted to showing that $|L| \geq(e(H)-1) b$.

In the proof of Corollary 1 in Section 4, we will show that if $H=P_{1}(k)$ for some $k \in \mathbb{P}$, then $T$ has at least $2 k b=(e(H)-1) b$ leaves. Therefore, assume $H$ consists of a central edge with $h$ leaves attached to one end and $k>h$ leaves attached to the other end, so that $(e(H)-1) b=(k+h) b$. Let $v \in V(T)$ be a vertex of maximum degree, and we consider the cases $d(v) \geq k b+1$ and $d(v) \leq k b$ separately.

Case 1. $d(v) \geq k b+1$.

If $h=1$, then $T \rightsquigarrow(H ; b)$ implies $T \rightsquigarrow\left(P_{3} ; b\right)$, so by Lemma 1 , there is a $w \neq v$ with $d(w) \geq b+1$, and therefore $|L| \geq(k b+1)+(b+1)-2=(k+1) b=(k+h) b$.

Otherwise $h>1$, so let $K_{1}, \ldots, K_{d(v)}$ be the connected components of $T-v$, and note that each contains a leaf of $T$. If $T\left[\{v\} \cup K_{i}\right] \not \leftrightarrow\left(S_{h+1} ; b\right)$ for all $i, 1 \leq i \leq d(v)$, we can color $T$ so that only $v$ sees at least $h+1$ colors. This contradicts the fact that $T \rightsquigarrow(H ; b)$, as $H$ has two vertices of degree at least $h+1$. Therefore $T\left[\{v\} \cup K_{i}\right] \rightsquigarrow\left(S_{h+1} ; b\right)$ for some $i$ and consequently has at least $h b+1$ leaves by Lemma 2 . This implies $K_{i}$ contains at least $h b$ leaves of $T$, and as the remaining components have at least one leaf of $T$ each, $|L| \geq d(v)-1+h b \geq(k+h) b$.

Case 2. $d(v) \leq k b$.

As in the proof of Lemma 2, color $T$ by rooting it at $v$ and coloring the edges between a vertex $u$ and its $\hat{d}(u)$ children using $\lceil\hat{d}(u) / b\rceil$ colors. Since a rainbow $H$ occurs, some vertex $w$ sees at least $k+1$ colors on incident edges, and $w \neq v$ since $\hat{d}(v) / b=d(v) / b \leq k$. Therefore $d(w) \geq(k-1) b+2$ and $d(v) \geq d(w)$, so

$$
|L| \geq 2((k-1) b+2)-2>2(k-1) b=(k+(k-2)) b,
$$

which suffices if $k-2 \geq h$, i.e. $k \geq h+2$.

Suppose now that $k=h+1$. If there is a $u \in V(T) \backslash\{v, w\}$ with $d(u) \geq b+1$, then

$$
|L| \geq 2((k-1) b+2)+(b+1)-2(2)>(2 k-1) b=(k+h) b,
$$


so we may assume $d(u) \leq b$ for all $u \in V(T) \backslash\{v, w\}$.

Let $(A, B)$ be a bipartition of $T$. If $v$ and $w$ lie in the same part, say, $A$, then every vertex in $B$ has degree at most $b$, and we can color the edges incident with each vertex in $B$ with a single color. But then $T \not \rightarrow\left(P_{3} ; b\right)$, a contradiction as $T \rightsquigarrow(H ; b)$ and $P_{3}$ is a subgraph of $H$. So, without loss of generality assume $v \in A$ and $w \in B$.

We now consider two further subcases, $v w \in E(T)$ and $v w \notin E(T)$ :

Case 2a. $v w \in E(T)$.

If $d(v)+d(w) \geq(2 k-1) b+2$, then $|L| \geq(2 k-1) b=(k+h) b$, so suppose otherwise. Then at most $(2 k-1) b$ edges in $T$ are incident with $v$ or $w$, so color $T$ as follows: first, root $T$ at $v$. For $u \in V(T) \backslash\{v, w\}, d(u) \leq b$, so color the edges between $u$ and its children with a single color. Since $d(v) \geq d(w) \geq(k-1) b+2$, color the edges between $w$ and $(k-1) b$ of its children with $k-1$ colors, and color the edges between $v$ and $(k-1) b$ of its children other than $w$ with $k-1$ colors. At most $(2 k-1) b-2(k-1) b=b$ edges remain uncolored, so color them with the same color. Then $v$ and $w$ each see $k$ colors on incident edges, and every $u \in V(T) \backslash\{v, w\}$ sees at most 2 colors. But then $T \not \leftrightarrow(H ; b)$, as no vertex has at least $k+1 \geq 3$ colors on incident edges, a contradiction.

Case 2b. $v w \notin E(T)$.

Let $x$ and $y$ be adjacent vertices on the path between $v$ and $w$ so that $v x \in E(T)$, $y \neq v$. Since $v \in A$ and $w \in B, d(v, w)$ is odd; since $d(v, y)=2$, therefore $y \neq w$. If $d(x)+d(y) \geq b+2$, then

$$
|L| \geq 2((k-1) b+2)+(b+2)-2(3)=(2 k-1) b=(k+h) b,
$$

so suppose otherwise. Therefore there are at most $b$ edges of $T$ incident with $x$ or $y$.

As $x$ has odd distance to $v$ and $y$ has odd distance to $w$, consider the tree $T-x y$. Let $\left(A^{*}, B^{*}\right)$ be a bipartition of $T-x y$ in which $x, y \in A^{*}$; so $v, w \in B^{*}$. Color all edges of $T$ incident with $x$ or $y$ with a single color, and for each $u \in A^{*} \backslash\{x, y\}$, color the edges incident with $u$ with a single color. Then $x y$ cannot lie on a rainbow $P_{3}$ in $T$, and by our earlier observation, $T-x y$ does not contain a rainbow $P_{3}$, therefore $T \not \leftrightarrow(H ; b)$.

\subsection{Clumps And Partial Coloring}

Suppose that $H$ and $T$ are trees, $e(H)>1$, and $T \rightsquigarrow(H ; b)$. Let $H^{\prime}=H[V(H) \backslash L(H)]$ and $T^{\prime}=T[V(T) \backslash L(T)]$, i.e. form $H^{\prime}$ and $T^{\prime}$ by removing the leaves of $H$ and $T$ respectively. For every $v \in V\left(T^{\prime}\right)$, let $L_{v}=N_{T}(v) \cap L(T)$.

We make two trivial observations about $T^{\prime}$ that will lead to very practical generalizations. The first observation is that no matter how we color the edges of $T$, no $x \in L(T)$ can correspond to any $y \in V\left(H^{\prime}\right)$ in any rainbow copy of $H$ in $T$, since $d_{T}(x)=1$ and $d_{H}(y)>1$. Since $T \rightsquigarrow(H ; b)$, this implies that $T^{\prime} \rightsquigarrow\left(H^{\prime} ; b\right)$. 
Our second observation is that the sets $L_{v}, v \in V\left(T^{\prime}\right)$, partition $V(T) \backslash V\left(T^{\prime}\right)$ and the trees $T\left[\{v\} \cup L_{v}\right], v \in V\left(T^{\prime}\right)$, partition the edges of $E(T) \backslash E\left(T^{\prime}\right)$, and consequently $e(T)=e\left(T^{\prime}\right)+\sum_{v \in V(T)}\left|L_{v}\right|$. Here as in the rest of the paper, we drop the restriction that all sets in a partition be nonempty.

To generalize our first observation, we introduce the following definition:

Definition 6. Let $H$ and $T$ be trees with $T \rightsquigarrow(H ; b)$, and let $X \subseteq V(H)$ and $S \subseteq V(T)$. We say that $S$ forbids $X$ if there is a coloring $f$ of the edges of $T$ incident with $S$ such that, under any extension of this coloring to all edges of $T$, no vertex in $S$ can correspond to any vertex in $X$ in any rainbow copy of $H$ in $T$. We call $f$ a forbidding coloring for $S$ with respect to $X$.

In our example earlier we see that $L(T)$ forbids $V\left(H^{\prime}\right)$, and any coloring of the edges incident with $L(T)$ is a forbidding coloring. As another example, suppose there is a $v \in V(T)$ with $d(v) \leq b$ : we can color the edges of $T$ incident with $v$ with a single color, as there are $d(v) \leq b$ of them, and consequently $\{v\}$ forbids $V\left(H^{\prime}\right)$.

We note that if $X \subseteq V(H)$, and $S_{1}, \ldots, S_{n}$ are disjoint subsets of $V(T)$ such that $e\left(S_{i}, S_{j}\right)=\left|\left\{a b \in E(T): a \in S_{i}, b \in S_{j}\right\}\right|=0$ for all $1 \leq i<j \leq n$, and each $S_{i}$ forbids $X$, then $S=\bigcup_{i=1}^{n} S_{i}$ forbids $X$.

To generalize our second observation, we introduce the following definition.

Definition 7. Let $T$ be a tree, and let $U$ be a subtree of $T$. Let $T-E(U)$ be the graph formed by removing the edges of $U$ from $T$, and for $v \in V(U)$, let $K_{v}$ denote the connected component of $T-E(U)$ containing $v$. Define

$$
C_{v}(U)=K_{v} \backslash\{v\}
$$

Now, rooting $T\left[K_{v}\right]$ at $v$, for all $w \in C_{v}(U)$ define

$$
C_{w}(U)=\{w\} \cup\left\{a \in K_{v}: a \text { is a descendant of } w\right\} .
$$

For all $v \in V(T)$, define $L_{v}(U)=C_{v}(U) \cap L(T)$.

We call the sets $C_{v}(U)$ clumps of $U$, and the vertices in $L_{v}(U)$ clumped leaves. When $U$ is understood from the context, we simply write $C_{v}$ and $L_{v}$.

In our earlier example, we have that for $v \in V\left(T^{\prime}\right), L_{v}=L_{v}\left(T^{\prime}\right)=C_{v}\left(T^{\prime}\right)$, and for $w \in V(T) \backslash V\left(T^{\prime}\right)$, we have $C_{w}\left(T^{\prime}\right)=\{w\}=L_{w}\left(T^{\prime}\right)$. We now state some basic properties of clumps. 


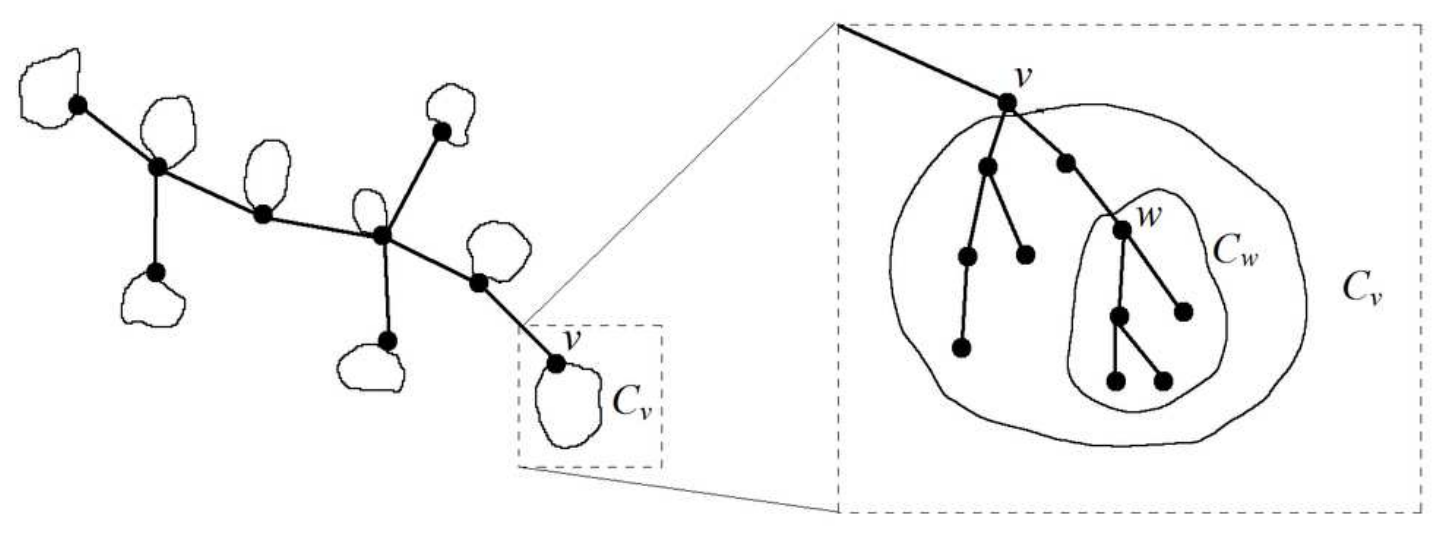

Figure 2: A subtree $U$ of a tree $T$, with $v \in V(U)$ and $w \in C_{v}$.

Lemma 4. Let $U$ be a subtree of a tree $T$. Then the following hold:

1. The sets $C_{v}=C_{v}(U), v \in V(U)$ form a partition (with possibly empty parts) of $V(T) \backslash V(U)$.

2. For any $x \in V(T),\left|C_{x}\right|$ is the number of edges of $T$ incident with vertices in $C_{x}$.

3. $e(T)=e(U)+\sum_{v \in V(U)}\left|C_{v}\right|$.

4. Let $z$ be a leaf of $U$, and let $y$ be its neighbor in $U$. Then $C_{y}(U-z)=C_{y}(U) \cup\{z\} \cup$ $C_{z}(U), C_{z}(U-z)=\{z\} \cup C_{z}(U)$, and $C_{x}(U-z)=C_{x}(U)$ for all $x \in V(T) \backslash\{y, z\}$.

5. If $U^{\prime}$ is a subtree of $U$, then $C_{v}\left(U^{\prime}\right) \supseteq C_{v}(U)$ for all $v \in V(U)$, and $C_{w}\left(U^{\prime}\right)=C_{w}(U)$ for all $w \in V(T) \backslash V(U)$.

Throughout much of the remainder of the paper, we will use clumps in the following manner: given $H$ and $T$ with $T \rightsquigarrow(H ; b)$, we will choose an appropriate subtree $H^{\prime}$ of $H$ such that $A R\left(H^{\prime} ; b\right)=G\left(H^{\prime} ; b\right)$. Then, we will construct a subtree $T^{\prime}$ of $T$ by enforcing conditions on the clumps $C_{v}\left(T^{\prime}\right)$ such that $C_{v}\left(T^{\prime}\right)$ forbids $V\left(H^{\prime}\right)$ for all $v \in V\left(T^{\prime}\right)$. In particular, this implies that $T^{\prime} \rightsquigarrow\left(H^{\prime} ; b\right)$, and by Lemma 4 we have $e(T)=e\left(T^{\prime}\right)+$ $\sum_{v \in V\left(T^{\prime}\right)}\left|C_{v}\left(T^{\prime}\right)\right| \geq G\left(H^{\prime} ; b\right)+\sum_{v \in V\left(T^{\prime}\right)}\left|C_{v}\left(T^{\prime}\right)\right|$, so all that will remain is to argue that $\sum_{v \in V\left(T^{\prime}\right)}\left|C_{v}\left(T^{\prime}\right)\right| \geq G(H ; b)-G\left(H^{\prime} ; b\right)$.

Our first application of these ideas provides a fairly general result on partial coloring. Suppose $H^{*}$ is a subtree of $H$, and $T \rightsquigarrow(H ; b)$. Then $T \rightsquigarrow\left(H^{*} ; b\right)$, so clearly any subset of $V(T)$ that forbids $H^{*}$ must exclude at least $A R\left(H^{*} ; b\right)+1$ vertices. Suppose now that we have found a subtree $U$ of $T$ so that $C_{v}(U)$ forbids $V\left(H^{*}\right)$ for every $v \in V(U)$. In the following lemma, we will show that there cannot be too many $x \in V(U)$ such that $\{x\} \cup C_{x}(U)$ forbids $V\left(H^{*}\right)$.

Lemma 5. Let $H$ be a tree, let $H^{*}$ be a subtree of $H$, and let $T \rightsquigarrow(H ; b)$. Let $U$ be a subtree of $T$ such that $C_{v}(U)$ forbids $V\left(H^{*}\right)$ with forbidding coloring $c_{v}$ for all $v \in V(U)$. Let $S_{0} \subset V(U), S_{0} \neq V(U)$, such that for all $x \in S_{0},\{x\} \cup C_{x}(U)$ forbids $V\left(H^{*}\right)$ with forbidding coloring $s_{x}$. Let $B_{0}=V(U) \backslash S_{0}$. 
Then $U$ contains a subtree $U^{\prime}$ such that $U^{\prime} \rightsquigarrow\left(H^{*} ; b\right),\left|V\left(U^{\prime}\right)\right| \leq\left|B_{0}\right|$, and for every $x \in S_{0} \cap V\left(U^{\prime}\right), d_{U^{\prime}}(x)<d_{U}(x)$. In particular, $\left|B_{0}\right| \geq A R\left(H^{*} ; b\right)+1$.

Proof. We assume that the colorings $c_{v}$ for $v \in V(U)$ and $s_{x}$ for $x \in S_{0}$ have pairwisedisjoint ranges. This can be done without loss of generality, as a forbidding coloring is determined by the edge-partition it induces via its color classes, and not the labels of the colors themselves. Moreover, as we will use the forbidding colorings to construct a partial coloring, this assumption will trivially allow us to maintain $b$-boundedness at each step of the construction.

We construct a partial coloring of $T$ as follows: Let $S=S_{0}, B=B_{0}$, and $C=\emptyset$. We note that $B_{0} \neq \emptyset$, and that for all $x \in S_{0}$, the edges of $T$ incident with $\{x\} \cup C_{x}(U)$ are the edges of $T\left[\{x\} \cup N_{U}(x) \cup C_{x}(U)\right]$.

While $S \neq \emptyset$,

1. Choose $x \in S$ with $N_{U}(x) \cap B \neq \emptyset$.

2. Apply $s_{x}$ to the edges of $T\left[\{x\} \cup N_{U}(x) \cup C_{x}(U)\right]$.

3. Let $B=B \cup N_{U}(x), S=S \backslash\left(\{x\} \cup N_{U}(x)\right)$, and $C=C \cup\{x\}$, and repeat.

First, we note that $B=B_{0} \neq \emptyset$ initially as $S \neq V(U)$, and $|B|$ never decreases. After each iteration, for each $x \in C, N_{U}(x) \subseteq B$ by Step 3. In particular, this means that if $S \neq \emptyset$, there is necessarily an $x \in S$ with $N_{U}(x) \cap B \neq \emptyset$, so Step 1 can be applied. Since $S \subseteq S_{0}$, Step 2 can be applied as well. By Step 3, $|S|$ decreases after each iteration, therefore the algorithm terminates with $B$ and $C$ partitioning $V(U)$ (with $C$ possibly empty).

For distinct $x, y \in C, x$ and $y$ are nonadjacent (as we set $N_{U}(x) \subseteq B$ ) and therefore the edges in $T\left[\{x\} \cup N_{U}(x) \cup C_{x}(U)\right]$ and $T\left[\{y\} \cup N_{U}(y) \cup C_{y}(U)\right]$ are disjoint, so no edge is colored more than once.

Claim 1. If $K$ is a connected component of $U-C,|K| \leq\left|B_{0}\right|$.

Proof of Claim 1. It suffices to show that after each iteration,

$$
\text { each component } K \text { of } U-C \text { satisfies }|K \cap B| \leq\left|B_{0}\right| \text {, }
$$

as every component of $U-C$ is a subset of $B$ after the final iteration.

Prior to the start of the algorithm, (2) holds trivially, so suppose it holds after $i \geq 0$ iterations, and $S \neq \emptyset$. Let $x \in S$ be the vertex chosen in Step 1 , and $K_{x}$ be the component of $T-C$ containing $x$. Since the only vertices whose labels change lie in $K_{x}$, we simply need to show (2) holds for each new component formed after Step 3, which we can identify with each $w \in N_{U}(x)$. Choose any such $w$, and let $K_{w}$ be the component of $U-(C \cup\{x\})$ containing $w$. Then prior to Step 3 we have

$$
\left.\left|\left(K_{w} \backslash\{w\}\right) \cap B\right|=\mid\left(K_{w} \backslash N_{U}(v)\right) \cap B\right)|\leq|\left(K_{x} \backslash N_{U}(x)\right) \cap B|\leq| K_{x} \cap B \mid-1,
$$

and after Step 3 we have $w \in B$, so $\left|K_{w} \cap B\right| \leq\left|K_{x} \cap B\right| \leq\left|B_{0}\right|$, and (2) holds after the $(i+1)$ st iteration. 
Until this point we have only colored edges incident with vertices in $\{x\} \cup C_{x}(U)$ for $x \in C$. For every $v \in B$, apply coloring $c_{v}$ to $T\left[\{v\} \cup C_{v}(U)\right]$. The only edges that remain uncolored are the edges of $U-C$. We consider an arbitrary coloring of these edges.

Since $T \rightsquigarrow(H ; b)$, there is a rainbow $H$ in $T$. Since $T\left[\{x\} \cup N_{U}(x) \cup C_{x}(U)\right]$ is colored according to $s_{x}$ for all $x \in C$, and $T\left[\{v\} \cup C_{v}(U)\right]$ is colored according to $c_{v}$ for all $v \in B$, the vertices corresponding to $V\left(H^{*}\right)$ cannot lie in $C \cup\left(\cup_{v \in V(U)} C_{v}(U)\right)$, and consequently must lie in $B=V(U) \backslash C$. In particular, some component of $U-C$ contains a rainbow copy of $H^{*}$, and since we can color the edges in each component independently, therefore some component $K$ of $U-C$ satisfies $T[K] \rightsquigarrow\left(H^{*} ; b\right)$. Let $U^{\prime}=T[K]$. Then $|V(U)|=|K| \leq\left|B_{0}\right|$ by Claim 1. Suppose $x \in V\left(U^{\prime}\right) \cap S_{0}$. Then $x \in V\left(U^{\prime}\right)$ implies $x \in B$, and consequently there is some $y \in C$ with $x y \in U$, and $y \notin V\left(U^{\prime}\right)$. Therefore, $d_{U^{\prime}}(x)<d_{U}(x)$.

Our first application of Lemma 5 will be the proof of Lemma 1.

Proof of Lemma 1. Let $n \geq 3, T \rightsquigarrow\left(P_{n} ; b\right)$, and $S_{0}=\{v \in V(T): d(v) \leq b\}$. Every $x \in S_{0}$ forbids the non-leaves of $P_{n}$, and every clump $C_{v}(T)=\emptyset$ for $v \in V(T)$. In other words, $\{x\} \cup C_{x}(T)$ forbids the non-leaves of $P_{n}$ for every $x \in S_{0}$.

If $S_{0}=V(T)$, then remove any vertex from $S_{0}$ arbitrarily, so that $\left|V(T) \backslash S_{0}\right|=1$. Applying Lemma 5, with $U=T, H=P_{n}, H^{*}=P_{n-2}$ and $S_{0}$,

$$
\left|V(T) \backslash S_{0}\right|=\left|V(U) \backslash S_{0}\right| \geq A R\left(H^{*} ; b\right)+1=A R\left(P_{n-2} ; b\right)+1 \geq 2 .
$$

In particular, we cannot have $\left|V(T) \backslash S_{0}\right|=1$, and therefore $V(T) \backslash S_{0}=\{v \in V(T)$ : $d(v) \geq b+1\}$, completing the proof.

With appropriate choices of the subtree $H^{*}$ of $H$ and of the subtree $U$ of $T$, we can force additional and useful structure on the $U^{\prime}$ produced by Lemma 5. An example of this is given in the following lemma.

Lemma 6. Let $H$ be a tree of diameter at least 3, and let $H^{\prime}$ be formed by removing all of the leaves of $H$, i.e. $H^{\prime}=H[V(H) \backslash L(H)]$. Let $h \in \mathbb{P}$ such that $d_{H}(v) \geq h+1$ for all $v \in L\left(H^{\prime}\right)$. Let $T \rightsquigarrow(H ; b)$. Then $T$ contains subtrees $U$ and $U^{\prime}$ with the following properties:

1. For all $u \in L(U),\left|L_{u}(U)\right| \geq h b$.

2. For all $v \in V(U), C_{v}(U)$ forbids $V\left(H^{\prime}\right)$, so $U \rightsquigarrow\left(H^{\prime} ; b\right)$.

3. $U^{\prime}$ is a subtree of $U$ and $U^{\prime} \rightsquigarrow\left(H^{\prime} ; b\right)$.

4. For all $v \in V\left(U^{\prime}\right), d_{U^{\prime}}(v) \geq b+1$ or $d_{U^{\prime}}+\left|L_{v}\left(U^{\prime}\right)\right| \geq h b+1$. 
Proof. Let $T \rightsquigarrow(H ; b)$. We will construct $U$ from $T$ so that Properties 1 and 2 hold, and then with an appropriate choice of $S_{0}$, apply Lemma 5 to construct $U^{\prime}$ and show that Properties 3 and 4 hold.

Claim 2. Let $R$ be a subtree of $T$, and let $v \in V(R)$ such that $1 \leq d_{R}(v) \leq b$ and $d_{R}(v)+\left|L_{v}(R)\right| \leq h b$. Then $\{v\} \cup C_{v}(R)$ forbids $V\left(H^{\prime}\right)$.

Proof of Claim 2. If $v$ is a leaf of $T$, the result follows trivially, so suppose otherwise. The edges of $T$ incident with vertices in $\{v\} \cup C_{v}(R)$ are the edges of $T\left[\{v\} \cup N_{R}(v) \cup C_{v}(R)\right]$, so for convenience let $\bar{T}=T\left[\{v\} \cup N_{R}(v) \cup C_{v}(R)\right]$. We note that as $v$ is not a leaf of $T$, the leaves of $\bar{T}$ are $N_{R}(v) \cup L_{v}(R)$, and $\left|N_{R}(v) \cup L_{v}(R)\right|=d_{R}(v)+\left|L_{v}(R)\right| \leq h b$.

If $h=1, \Delta(\bar{T}) \leq b$, so let $(A, B)$ be a bipartition of $\bar{T}$ with $v \in A$, and color each edge $e \in E(\bar{T})$ with the color $e \cap A$. Then $\bar{T}$ does not contain a rainbow $P_{3}$ and consequently no rainbow $H$, and all edges incident with $v$ have the same color. Extending this to a coloring of all of $T$, we note that $v$ can only serve as a leaf in a rainbow copy of $H$, and the result follows.

If $h>1$, then let $x \in V(\bar{T}) \backslash N_{R}(v)$ with $d_{\bar{T}}(x)=\Delta(\bar{T})$ : as vertices in $N_{R}(v)$ are leaves in $\bar{T}$ and $v \in V(\bar{T}) \backslash N_{R}(v)$, such an $x$ exists. Then, by the proof of Lemma 2 , if we root $\bar{T}$ at $x$ and color the edges between a vertex $u \in V(\bar{T})$ and its children using $\lceil\hat{d}(u) / b\rceil$ colors, $\bar{T}$ contains no rainbow $S_{h+1}$. As $x \notin N_{R}(v)$, vertices in $N_{R}(v)$ are children of $v$ and $\left|N_{R}(v)\right| \leq b$, we may assume that the edges between $v$ and $N_{R}(v)$ have the same color. Extend this to a coloring of $T$, and consider a rainbow copy of $H$ in $T$ : the vertices corresponding to $L\left(H^{\prime}\right)$ cannot lie in $\{v\} \cup C_{v}(R)$. If $v$ corresponds to a vertex in $V\left(H^{\prime}\right) \backslash L\left(H^{\prime}\right)$, since the edges between $v$ and $N_{R}(v)$ have the same color, exactly one such edge can be used, implying $C_{v}(R)$ contains a vertex corresponding to one in $L\left(H^{\prime}\right)$, a contradiction.!

Now, let $T_{0}=T, i=0$, and if $T_{i}$ contains a leaf $v_{i}$ with $\left|L_{v_{i}}\left(T_{i}\right)\right|<h b$, define $T_{i+1}=T_{i}-v_{i}$, increment $i$, and repeat. This process terminates with a tree $T_{k}, k \leq e(T)$, so let $U=T_{k}$. Note that Property 1 holds by construction, so we show Property 2 holds as well.

Claim 3. $C_{v}(U)$ forbids $V\left(H^{\prime}\right)$ for all $v \in V(U)$.

Proof of Claim 3. Since the $C_{w}(U)$ where $w \in N(v) \backslash V(U)$ partition $C_{v}(U)$, and the edges of $T$ incident with each such $C_{w}(U)$ are the edges in $T\left[\{v\} \cup C_{w}(U)\right]$, if each $C_{w}(U)$ forbids $V\left(H^{\prime}\right)$ then $C_{v}(U)$ does as well.

Let $w \in N(v) \backslash V(U)$. Then $w=v_{i}$ for some $i, 0 \leq i \leq k-1$, so $w$ is a leaf of $T_{i}$, $\left|L_{w}\left(T_{i}\right)\right|<h b$ and $T_{i+1}=T_{i}-w$. Since $d_{T_{i}}(w)+\left|L_{w}\left(T_{i}\right)\right| \leq h b$, by Claim $2\{w\} \cup C_{w}\left(T_{i}\right)$ forbids $V\left(H^{\prime}\right)$. By Lemma $4, C_{w}\left(T_{i+1}\right)=C_{w}\left(T_{i}-w\right)=\{w\} \cup C_{w}\left(T_{i}\right)$, and as $U$ is a subtree of $T_{i+1}, C_{w}(U)=C_{w}\left(T_{i+1}\right)=\{w\} \cup C_{w}\left(T_{i}\right)$, so $C_{w}(U)$ forbids $V\left(H^{\prime}\right)$.

Claim 3 shows that $U \rightsquigarrow\left(H^{\prime} ; b\right)$, and therefore $e(U) \geq e\left(H^{\prime}\right) \geq 1$ as $H$ has diameter at least 3. Consequently, $L(U) \neq \emptyset$. Let $S=\left\{x \in V(U): d_{U}(x)+\left|L_{x}(U)\right| \leq h b\right\}$. 
By Claim 2, for every $x \in S,\{x\} \cup C_{x}(U)$ forbids $V\left(H^{\prime}\right)$. As $S \cap L(U)=\emptyset$, therefore $S \subsetneq V(U)$, and applying Lemma 5 with $S_{0}=S$, we see that $U$ contains a subtree $U^{\prime}$ such that $U^{\prime} \rightsquigarrow\left(H^{\prime} ; b\right)$ and $d_{U^{\prime}}(x)<d_{U}(x)$ for all $x \in S \cap V\left(U^{\prime}\right)$. Consequently, Property 3 holds. All that remains is to show Property 4 holds.

Claim 4. For all $v \in V\left(U^{\prime}\right), d_{U^{\prime}}(v) \geq b+1$ or $d_{U^{\prime}}(v)+\left|L_{v}\left(U^{\prime}\right)\right| \geq h b+1$.

Proof of Claim 4. Let $v \in V\left(U^{\prime}\right)$ with $d_{U^{\prime}}(v) \leq b$, and first suppose that $d_{U^{\prime}}(v)=d_{U}(v)$. Then $v \notin S$, and consequently $d_{U}(v)+\left|L_{v}(U)\right| \geq h b+1$. But $L_{v}(U) \subseteq C_{v}(U)$, and as $U^{\prime}$ is a subtree of $U$, by Lemma $4, L_{v}(U) \subseteq L_{v}\left(U^{\prime}\right)$, so $d_{U^{\prime}}(v)+\left|L_{v}\left(U^{\prime}\right)\right| \geq d_{U}(v)+\left|L_{v}(U)\right| \geq h b+1$.

If $d_{U^{\prime}}(v)<d_{U}(v)$, consider the forest $U-E\left(U^{\prime}\right): d_{U}(v)>d_{U^{\prime}}(v)$ implies $v$ lies in some component $K$ of $U-E\left(U^{\prime}\right)$ with $|K|>1$, and as $U^{\prime}$ is a subtree of $U, K \cap L(U) \neq \emptyset$. Letting $u \in K \cap L(U)$ and noting that $U-E\left(U^{\prime}\right)$ is a subgraph of $T-E\left(U^{\prime}\right)$, therefore $u \in C_{v}\left(U^{\prime}\right)$. But, by Lemma $4, L_{u}(U) \subseteq C_{u}(U) \subseteq C_{u}\left(U^{\prime}\right) \subseteq C_{v}\left(U^{\prime}\right)$, which immediately implies $\left|L_{v}\left(U^{\prime}\right)\right| \geq\left|L_{u}(U)\right| \geq h b$, and $d_{U^{\prime}}(v)+\left|L_{v}\left(U^{\prime}\right)\right| \geq h b+1$ follows.

\section{Proof of Theorem 1}

By Corollary 2 and Lemmas 2 and 3, Conjecture 1 is true for trees of diameter at most 3, so we assume $H$ has diameter 4 . Letting $\hat{H}=H[V(H) \backslash L(H)]$, we see $\hat{H}$ has diameter 2 and is therefore an $S_{n}$ for some $n \geq 2$. Let $v_{0}$ denote the central vertex of $\hat{H}$, and $v_{1}, v_{2}, \ldots, v_{n}$ denote its leaves, such that $d_{H}\left(v_{1}\right) \geq d_{H}\left(v_{2}\right) \geq \cdots \geq d_{H}\left(v_{n}\right)$. Let $k_{i}=d_{H}\left(v_{i}\right)-1$ for $1 \leq i \leq n$ and $k_{0}=d_{H}\left(v_{0}\right)-n$, so that $k_{i}$ is the number of leaves adjacent to $v_{i}$ in $H$. By our construction, $k_{1} \geq k_{2} \geq \cdots \geq k_{n} \geq 1$.

Our goal is to show that

$$
A R(H ; b)=F\left(H, v_{0} v_{1} ; b\right)=\left(\sum_{i=2}^{n} k_{i}\right) b^{2}+\left(k_{1}+k_{0}+n-1\right) b+1
$$

We will show this by induction on $n+k_{1}$, but the case $n=2$ poses a more technical challenge than when $n>2$. As such, we state it as a lemma and prove it in Section 3.1.

Lemma 7. If $H$ is as above with $n=2$, then

$$
A R(H ; b)=F\left(H, v_{0} v_{1} ; b\right)=k_{2} b^{2}+\left(k_{0}+k_{1}+1\right) b+1
$$

We now suppose that $n \geq 3$. Let $H^{\prime}$ be the tree formed by removing a leaf adjacent with each of $v_{1}, \ldots, v_{n}$. As this removes one edge adjacent with $v_{0} v_{1}$ and $(n-1)$ edges of distance 2 from $v_{0} v_{1}$ in the line graph $\mathcal{L}(H)$, we have

$$
F\left(H^{\prime}, v_{0} v_{1} ; b\right)=F\left(H, v_{0} v_{1} ; b\right)-\left((n-1) b^{2}+b\right) .
$$


Claim 5. $A R\left(H^{\prime} ; b\right)=F\left(H^{\prime}, v_{0} v_{1} ; b\right)$.

Proof of Claim 5. Suppose first that $k_{1}=1$. Then $1=k_{1} \geq \cdots \geq k_{n} \geq 1$ implies equality holds throughout, and consequently $d_{H^{\prime}}\left(v_{i}\right)=1$ for $1 \leq i \leq n$, so $H^{\prime}$ is a star, and as $v_{0} v_{1}$ is an edge of $H^{\prime}$, by Lemma 2 , therefore $A R\left(H^{\prime} ; b\right)=F\left(H^{\prime}, v_{0} v_{1} ; b\right)$.

If $k_{1}>1$ and $k_{2}=1$, then by an analogous argument, $H^{\prime}$ is a tree of diameter 3 with central edge $v_{0} v_{1}$, so by Lemma $3, A R\left(H^{\prime} ; b\right)=F\left(H^{\prime}, v_{0} v_{1} ; b\right)$.

Finally, if $k_{1}>1$ and $k_{2}>1$, then $H^{\prime}$ has diameter 4 , so consider $\hat{H}^{\prime}=H^{\prime}\left[V\left(H^{\prime}\right) \backslash\right.$ $\left.L\left(H^{\prime}\right)\right]$. As $d_{H^{\prime}}\left(v_{i}\right)=k_{i}-1$ for $1 \leq i \leq n$, and $k_{1}, k_{2}>1, \hat{H}^{\prime}$ is an $S_{n^{\prime}}$ for some $n^{\prime}$, $2 \leq n^{\prime} \leq n$ with central vertex $v_{0}$ and leaves $v_{1}, \ldots, v_{n^{\prime}}$, and $d_{H^{\prime}}\left(v_{1}\right) \geq \cdots \geq d_{H^{\prime}}\left(v_{n^{\prime}}\right)$. Letting $k_{1}^{\prime}=d_{H^{\prime}}\left(v_{1}\right)-1=k_{1}-1$, we therefore have $n^{\prime}+k_{1}^{\prime} \leq n+k-1$, and by induction $A R\left(H^{\prime} ; b\right)=F\left(H^{\prime}, v_{0} v_{1} ; b\right)$.

Now, let $T \rightsquigarrow(H ; b)$.

Claim 6. $T$ contains a subtree $T^{\prime}$ such that $T^{\prime} \rightsquigarrow\left(H^{\prime} ; b\right)$, and $e(T)-e\left(T^{\prime}\right) \geq(n-1) b^{2}+b$.

Proof of Claim 6. Applying Lemma 6 to $H$ and $T$ with $h=1$, we find a subtree $\hat{T}$ of $T$ such that $\hat{T} \rightsquigarrow(\hat{H} ; b)$ and for all $v \in V(\hat{T}), d_{\hat{T}}(v) \geq b+1$ or $d_{\hat{T}}(v)+\left|L_{v}(\hat{T})\right| \geq b+1$. Since $\hat{H}$ is an $S_{n}$ with $n \geq 3$, by Lemma 2 we have $|L(\hat{T})| \geq(n-1) b+1$, and for all $u \in L(\hat{T})$, we have $\left|L_{u}(\hat{T})\right| \geq b$, and consequently $\left|C_{u}(\hat{T})\right| \geq b$.

We now construct a partial coloring of $T$ : For each $u \in L(\hat{T})$, root the tree $T[\{u\} \cup$ $\left.C_{u}(\hat{T})\right]$ at $u$. Color edges of maximum depth using a single color $\alpha_{u}$ until $b$ edges are colored (which is possible as $\left|C_{u}(\hat{T})\right| \geq b$ ).

Suppose $x y$ is a colored edge in $T\left[\{u\} \cup C_{u}(\hat{T})\right]$, where $x$ is the parent of $y$. By construction, all edges of $T$ incident with $y$ have color $\alpha_{u}$, so $\{y\}$ forbids $V(\hat{H})$. For each child $z$ of $x$, any edge of $T$ incident with $z$ other than $x z$ necessarily has color $\alpha_{u}$. In particular, this implies that $x$ cannot be the endpoint of three color-disjoint rainbow $P_{2}$ 's in $T$ under any extension, by noting that $x \in C_{u}(\hat{T})$ implies $x$ has a unique parent, and $x=u$ implies $x$ has a unique neighbor outside $C_{u}(\hat{T})$ as $u \in L(\hat{T})$.

Therefore $\{x\}$ forbids $\left\{v_{0}\right\}$ under this coloring, so if $x y$ is an edge of a rainbow $H$ under some extension, then $x$ corresponds to some $v_{i}, 1 \leq i \leq n$. Additionally, edges with different colors are nonadjacent under this coloring.

Letting $T^{\prime}$ be the tree formed by removing the colored edges (as we colored from maximum depth in each clump, $T^{\prime}$ is a tree), we conclude that $T^{\prime} \rightsquigarrow\left(H^{\prime} ; b\right)$, and $e(T)-$ $e\left(T^{\prime}\right)=b|L(\hat{T})| \geq(n-1) b^{2}+b$.

Combining Claims 5 and 6 with (4), therefore

$$
\begin{aligned}
e(T) & \geq e\left(T^{\prime}\right)+(n-1) b^{2}+b \\
& \geq A R\left(H^{\prime} ; b\right)+(n-1) b^{2}+b \\
& =F\left(H^{\prime}, v_{0} v_{1} ; b\right)+(n-1) b^{2}+b \\
& =F\left(H, v_{0} v_{1} ; b\right),
\end{aligned}
$$


completing the proof.

\subsection{Proof of Lemma 7}

To simplify notation, let $l=k_{0}, k=k_{1}, h=k_{2}$, and let $v_{l}, v_{k}, v_{h}$ denote $v_{0}, v_{1}, v_{2}$. Let $T \rightsquigarrow(H ; b)$. Our goal is to show that

$$
e(T) \geq F\left(H, v_{l} v_{k} ; b\right)=h b^{2}+(k+l+1) b+1 .
$$

By Lemma 6, there is a subtree $T^{\prime}$ of $T$ so that $T^{\prime} \rightsquigarrow\left(P_{2} ; b\right)$, and for all $v \in V\left(T^{\prime}\right)$, $d_{T^{\prime}}(v) \geq b+1$ or $d_{T^{\prime}}(v)+\left|L_{v}\left(T^{\prime}\right)\right| \geq h b+1$.

We first mention that if $e\left(T^{\prime}\right)>b+1$ and $z \in L\left(T^{\prime}\right)$, then $T^{\prime}-z$ satisfies the same conditions: since $e\left(T^{\prime}-z\right) \geq b+1, T^{\prime}-z \rightsquigarrow\left(P_{2} ; b\right)$. Letting $y$ be $z$ 's neighbor in $T^{\prime}$, we have $C_{y}\left(T^{\prime}-z\right) \supseteq C_{z}\left(T^{\prime}\right)$ and $C_{x}\left(T^{\prime}-z\right)=C_{x}\left(T^{\prime}\right)$ for all $x \in V\left(T^{\prime}\right) \backslash\{y, z\}$ by Lemma 4 . Since $d_{T^{\prime}-z}(x)=d_{T^{\prime}}(x)$ for $x \in V\left(T^{\prime}\right) \backslash\{y, z\}$, it suffices to note that $d_{T^{\prime}-z}(y)+\left|L_{y}\left(T^{\prime}-z\right)\right| \geq 1+\left|L_{z}\left(T^{\prime}\right)\right| \geq 1+h b$. In particular, we can assume $e\left(T^{\prime}\right)=b+1$ without loss of generality.

While our goal is to show that $e(T)$ is sufficiently large, our decision to reduce $e\left(T^{\prime}\right)$ to $b+1$ (if necessary) is that it provides us with an advantage when constructing partial colorings. Specifically, if $R$ is any tree of size $b+1$ and $r \in V(R) \backslash L(R)$, then we can color the edges of $R$ using two colors so that vertex $r$ is the center of every rainbow $P_{2}$ in $R$ produced by this coloring. This can easily be shown by induction on $b$.

We also mention that for the arguments that follow, we will only need the condition $d_{T^{\prime}}(v)+\left|C_{v}\left(T^{\prime}\right)\right| \geq h b+1$ to hold for $v \in V\left(T^{\prime}\right)$ with $d_{T^{\prime}}(v) \leq b$, which trivially does as $L_{v}\left(T^{\prime}\right) \subseteq C_{v}\left(T^{\prime}\right)$ for all $v \in V\left(T^{\prime}\right)$.

As we will only deal with clumps of $T^{\prime}$ from this point on, we will simply write $C_{v}$ instead of $C_{v}\left(T^{\prime}\right)$, and we will use $d^{\prime}$ in place of $d_{T^{\prime}}$ and $V^{\prime}$ in place of $V\left(T^{\prime}\right)$ to further minimize notation. In what follows, we will use the conditions on $T^{\prime}$ to construct a partial coloring of $T$ that yields the quadratic term in (5), and then argue that the uncolored edges remaining must make up the linear gap.

Claim 7. Let $v \in V\left(T^{\prime}\right) \backslash L\left(T^{\prime}\right)$, and suppose $\left|C_{x}\right| \geq h b$ for all $x \in V^{\prime} \backslash\{v\}$. Then (5) holds.

Proof of Claim 7. For every $x \in V^{\prime} \backslash\{v\}$, color (any) $h b$ edges incident with vertices in $C_{x}$ using $h$ colors. This colors $h b(b+1)=h b^{2}+h b$ edges in total, so let $\gamma=e(T)-\left(h b^{2}+h b\right)$ be the number of uncolored edges remaining. Note that all edges incident with vertices in $C_{v}$ are uncolored, as are the edges of $T^{\prime}$.

By way of contradiction, suppose $\gamma \leq(k+l+1-h) b$. Then we can color the remaining edges using at most $k+l+1-h$ colors. Also, as $e\left(T^{\prime}\right)=b+1$ and $d(v) \geq 2$, we can do so by first coloring the edges of $T^{\prime}$ using two colors so $v$ is the center of every rainbow $P_{2}$ in $T^{\prime}$. Since $T \rightsquigarrow(H ; b)$, a rainbow copy of $H$ occurs: let $u_{h}, u_{l}, u_{k}$ be the vertices of $T$ corresponding to $v_{h}, v_{l}, v_{k}$, respectively, in that copy. 
First, we see that $\left\{u_{h}, u_{l}, u_{k}\right\} \nsubseteq\{x, y\} \cup C_{x} \cup C_{y}$ for any edge $x y$ of $T^{\prime}$, as the edges incident with vertices in $\{x, y\} \cup C_{x} \cup C_{y}$ have at most $2 h+(k+l+1-h)=k+l+h+1=$ $e(H)-1$ colors on them, and edges incident with $\left\{u_{h}, u_{l}, u_{k}\right\}$ have at least $e(H)$ colors.

This implies that $\left\{u_{h}, u_{l}, u_{k}\right\} \subseteq V^{\prime}$, and by our coloring of the edges in $T^{\prime}$, we must have $u_{l}=v$. But the edges incident with vertices in $\left\{u_{h}, u_{k}, v\right\} \cup C_{u_{h}} \cup C_{u_{k}} \cup C_{v}$ again have at most $2 h+(k+l+1-h)=e(H)-1$ colors on them, a contradiction. Therefore $\gamma \geq(k+l+1-h) b+1$, and (5) follows.

Suppose that $\Delta\left(T^{\prime}\right)=b+1$. Then $e\left(T^{\prime}\right)=b+1$ implies that $T^{\prime}$ is a star with central vertex $v$, and $V^{\prime} \backslash\{v\}=L\left(T^{\prime}\right)$. But for all $x \in L\left(T^{\prime}\right), d^{\prime}(x)=1 \leq b$, so $\left|C_{x}\right| \geq h b+1-d^{\prime}(v)=h b$, and by Claim 7, (5) holds.

Therefore, we assume $\Delta\left(T^{\prime}\right) \leq b$, and consequently $d^{\prime}(v)+\left|C_{v}\right| \geq h b+1$ for all $v \in V^{\prime}$. By Lemma 4,

$$
e(T)=e\left(T^{\prime}\right)+\sum_{v \in V^{\prime}}\left|C_{v}\right| \geq(b+1)+\sum_{v \in V^{\prime}}\left(h b+1-d^{\prime}(v)\right)=h b^{2}+2 h b+1,
$$

so if $2 h \geq k+l+1,(5)$ follows immediately.

Suppose instead that $k+l+1>2 h$. As we can rewrite that as $(k-h)+(l-(h-1))>0$, we separate the remainder of the proof into two cases: $l \leq h-1$ and $l \geq h$.

Case 1. $l \leq h-1$.

For each $v \in V^{\prime}$, color $\min \left\{\left|C_{v}\right|, h b\right\}$ edges incident with vertices in $C_{v}$ using at most $h$ colors. Since $d^{\prime}(v)+\left|C_{v}\right| \geq h b+1$ and $d^{\prime}(v) \geq 1$, at least

$$
\sum_{v \in V^{\prime}}\left(h b+1-d^{\prime}(v)\right)=(h b+1)(b+2)-2(b+1)=h b^{2}+(2 h-1) b
$$

edges are colored, and every vertex sees at most $h$ colors on colored incident edges. Let $\gamma$ be the number of uncolored edges remaining in $T$.

Suppose $\gamma \leq(k-h+1) b$. Color the remaining edges using at most $k-h+1$ colors. Since $T$ contains a rainbow copy of $H$ under this coloring, let $u_{k}$ and $u_{h}$ denote the vertices corresponding to $v_{k}$ and $v_{h}$ in that copy. Since $v_{k}$ and $v_{h}$ are nonadjacent in $H$ and $d_{H}\left(v_{k}\right)+d_{H}\left(v_{h}\right)=k+h+2$, the edges incident with $u_{h}$ and $u_{k}$ must have at least $k+h+2$ colors on them. But, by our coloring, $u_{h}$ and $u_{k}$ see at most $2 h+(k-h+1)=h+k+1$ colors on incident edges, a contradiction. Therefore $\gamma \geq(k-h+1) b+1$, and

$$
e(T) \geq\left(h b^{2}+(2 h-1) b\right)+\gamma \geq h b^{2}+(k+h) b+1 \geq h b^{2}+(k+l+1) b+1 .
$$

Case 2. $l \geq h$.

If $\left|C_{x}\right| \geq h b$ for all $x \in V^{\prime}$, then Claim 7 yields the result, so suppose otherwise. Let $v \in V^{\prime}$ with $\left|C_{v}\right|<h b$; note that $v$ is not a leaf of $T^{\prime}$. Then $\left|C_{v}\right|+e\left(T^{\prime}\right) \leq(h+1) b$ and 
we can color the edges of $T\left[V^{\prime} \cup C_{v}\right]$, i.e. those in $T^{\prime}$ and those incident with vertices in $C_{v}$, using $h+1$ colors. In particular, we can assume that the edges of $T^{\prime}$ have only two colors, and $v$ is the center of every rainbow $P_{2}$ in $T^{\prime}$.

For each $x \in V^{\prime} \backslash\{v\}$, color $\min \left\{\left|C_{x}\right|, h b\right\}$ edges incident with vertices in $C_{x}$ using at most $h$ colors. Then for every edge $y z \in E\left(T^{\prime}\right)$, the edges of $T$ incident with vertices in $\{y, z\} \cup C_{y} \cup C_{z}$ have at most $2 h+1$ colors on them. Additionally, we have colored at least $\sum_{y \in V^{\prime}}\left(h b+1-d^{\prime}(y)\right)+b+1=h b^{2}+2 h b+1$ edges of $T$. Let $\gamma$ be the number of edges that remain uncolored.

Suppose $\gamma \leq(k+l-h) b$ : color the remaining edges of $T$ using at most $k+l-h$ colors. Let $u_{h}, u_{l}, u_{k}$ be the vertices corresponding to $v_{h}, v_{l}, v_{k}$ in a rainbow copy of $H$ in $T$. Since every rainbow $P_{2}$ in $T^{\prime}$ has central vertex $v$, and the edges incident with $v$ have been colored with at most $h+1$ colors, we see that $u_{l} \neq v$, as $d_{H}\left(v_{l}\right)=l+2>h+1$. Consequently, $\left\{u_{h}, u_{l}, u_{k}\right\} \subseteq\{y, z\} \cup C_{y} \cup C_{z}$ for some edge $y z$ of $T^{\prime}$. But the edges of $T$ incident with vertices in $\{y, z\} \cup C_{y} \cup C_{z}$ have at most $(2 h+1)+(k+l-h)=k+l+h+1=e(H)-1$ colors on them, a contradiction.

Therefore $\gamma \geq(k+l-h) b+1$, and

$e(T) \geq h b^{2}+2 h b+1+(k+l-h) b+1>h b^{2}+(k+l+h) b+1 \geq h b^{2}+(k+l+1) b+1$.

\section{Proofs of Theorem 2 and Corollary 1}

Since $H \in \mathcal{S}_{b}$, there is an $e \in E(H)$ such that $A R(H, b)=F(H, e ; b)$ and for every tree $U$ with $U \rightsquigarrow(H ; b), U$ has at least $L(H, e ; b)$ edge leaves. Our goal will be to show that $A R(H(k) ; b)=F(H(k), e ; b)$ and for every tree $T$ with $T \rightsquigarrow(H(k) ; b), T$ has at least $L(H(k), e ; b)$ leaves.

Claim 8. $L(H(k), e ; b)=k b \cdot L(H, e ; b)$ and $F(H(k), e ; b)=F(H, e ; b)+k b \cdot L(H, e ; b)$.

Proof of Claim 8. Recall that $L_{H}$ and $L_{H(k)}$ are the sets of edge leaves of $H$ and $H(k)$ respectively. As $e(H) \geq 2$, there is a natural bijection between $L_{H}$ and $L(H)$, namely $f(e)=v$ where $v \in e \cap L(H)$. As $H(k)$ is formed by adding $k$ edge leaves to each vertex leaf of $H, L_{H(k)}=E(H(k)) \backslash E(H)$. Moreover, for each $g \in L_{H(k)}$ there is a unique $f \in L_{H}$ such that $f$ and $g$ are adjacent, and for each $f \in L_{H}$ there are exactly $k$ distinct $g \in L_{H(k)}$ with $f$ and $g$ adjacent. Consequently, for each $f \in L_{H}$ and $g \in L_{H(k)}$ with $f$ and $g$ adjacent, $d(e, g)=d(e, f)+1$ as $g \notin E(H)$.

Therefore

$$
L(H(k), e ; b)=\sum_{g \in L_{H(k)}} b^{d(e, g)}=\sum_{f \in L_{H}} k \cdot b^{d(e, f)+1}=k b \sum_{f \in L_{H}} b^{d(e, f)}=k b \cdot L(H, e ; b),
$$


and

$$
\begin{aligned}
F(H(k), e ; b) & =\sum_{f \in E(H(k))} b^{d(e, f)} \\
& =\sum_{f \in E(H)} b^{d(e, f)}+\sum_{g \in L_{H(k)}} b^{d(e, g)} \\
& =F(H, e ; b)+k b \cdot L(H, e ; b) .
\end{aligned}
$$

Suppose $T \rightsquigarrow(H(k) ; b)$. As $e(H) \geq 2, H(k)$ has diameter at least 3. Applying Lemma 6 , there is a subtree $T^{\prime}$ of $T$ with $T^{\prime} \rightsquigarrow(H ; b)$ and for all $v \in V\left(T^{\prime}\right), d_{T^{\prime}}(v) \geq b+1$ or $d_{T^{\prime}}(v)+\left|L_{v}\left(T^{\prime}\right)\right| \geq k b+1$.

As $e\left(T^{\prime}\right) \geq e(H) \geq 2$ and $H \in \mathcal{S}_{b}$, therefore $\left|L\left(T^{\prime}\right)\right| \geq L(H, e ; b)$. For each $v \in$ $L\left(T^{\prime}\right), d_{T^{\prime}}(v)=1$ implies $\left|L_{v}\left(T^{\prime}\right)\right| \geq k b$, and consequently $|L(T)| \geq \sum_{v \in L\left(T^{\prime}\right)}\left|L_{v}\left(T^{\prime}\right)\right| \geq$ $L(H, e ; b) \cdot k b=L(H(k), e ; b)$, so $T$ has at least $L(H(k), e ; b)$ vertex leaves, and consequently at least that many edge leaves. Finally,

$$
e(T) \geq e\left(T^{\prime}\right)+\sum_{v \in V\left(T^{\prime}\right)}\left|L_{v}\left(T^{\prime}\right)\right| \geq F(H, e ; b)+k b \cdot L(H, e ; b)=F(H(k), e ; b),
$$

so $A R(H(k) ; b)=F(H(k), e ; b)$ and therefore $H(k) \in \mathcal{S}_{b}$.

Proof of Corollary 1. Corollary 2 and Lemma 2 show that $H \in \mathcal{S}$ if $H=P_{n}$ or $H=S_{n}$ for $n \geq 3$, and clearly $P_{0}, P_{1} \in \mathcal{S}$. Suppose $H=P_{a}(k)$, with $a \in\{1,2\}$ and $k \in \mathbb{P}$.

Let $T \rightsquigarrow(H ; b)$. As $H$ has diameter at least 3 and the tree formed by removing all leaves of $H$ is $P_{a}$, by Lemma 6 there is a subtree $T^{\prime}$ of $T$ such that $T^{\prime} \rightsquigarrow\left(P_{a} ; b\right)$ and $d_{T^{\prime}}(v) \geq b+1$ or $d_{T^{\prime}}(v)+\left|L_{v}\left(T^{\prime}\right)\right| \geq k b+1$ for all $v \in V\left(T^{\prime}\right)$. Again, for notational convenience we will write $d^{\prime}, V^{\prime}, E^{\prime}$ for $d_{T^{\prime}}, V\left(T^{\prime}\right)$, and $E\left(T^{\prime}\right)$, respectively. We only consider clumps of $T^{\prime}$, so we will just write $C_{v}$ and $L_{v}$.

If $a=1$, let $e$ denote the center edge of $H . T^{\prime} \rightsquigarrow\left(P_{1} ; b\right)$ implies $T^{\prime}$ has at least 2 leaves, say $u_{1}$ and $u_{2}$, and $d^{\prime}\left(u_{1}\right)=d^{\prime}\left(u_{2}\right)=1$ implies $\left|L_{u_{1}}\right| \geq k b$ and $\left|L_{u_{2}}\right| \geq k b$. Therefore $|L(T)| \geq\left|L_{u_{1}}\right|+\left|L_{u_{2}}\right|=2 k b=L(H, e ; b)$, and each vertex leaf corresponds to a unique edge leaf. Since $e(T) \geq 1+2 k b=F(H, e ; b)$ follows immediately, therefore $H \in \mathcal{S}_{b}$, and as $b$ is arbitrary, therefore $H \in \mathcal{S}$. This completes the proof of Lemma 3, so trees of diameter 3 lie in $\mathcal{S}$.

If $a=2$, letting $e$ be either edge on the interior $P_{2}$ of $H$, then $A R(H ; b)=F(H, e ; b)$ by Lemma 7. By our earlier comments it suffices to show $|L(T)| \geq L(H, e ; b)$, and direct computation gives $L(H, e ; b)=k b(b+1)$. 
If $\Delta\left(T^{\prime}\right)=b+1,\left|L\left(T^{\prime}\right)\right| \geq b+1$, and consequently

$$
|L(T)| \geq \sum_{u \in L\left(T^{\prime}\right)}\left|L_{u}\right| \geq k b\left|L\left(T^{\prime}\right)\right| \geq k b(b+1) .
$$

Otherwise, $d^{\prime}(v)+\left|L_{v}\right| \geq k b+1$ for all $v \in V^{\prime}$, and therefore

$$
\begin{aligned}
|L(T)| & \geq \sum_{v \in V^{\prime}}\left|L_{v}\right| \\
& \geq \sum_{v \in V^{\prime}}\left(k b+1-d^{\prime}(v)\right) \\
& =(k b+1)\left|V^{\prime}\right|-2\left|E^{\prime}\right| \\
& =(k b-1)\left|E^{\prime}\right|+k b+1 \\
& \geq(k b-1)(b+1)+k b+1 \\
& =k b(b+1)+(k-1) b,
\end{aligned}
$$

as $\left|E^{\prime}\right| \geq A R\left(P_{2} ; b\right)=b+1$. Since $k \in \mathbb{P},(k-1) b \geq 0$ and therefore $H \in \mathcal{S}_{b}$, and as $b$ is arbitrary, therefore $H \in \mathcal{S}$.

\section{$5 \quad$ Proof of Theorem 3}

First, we note that if $n=2 r-1$, letting $e$ be the centermost edge on a longest path of $H$ gives $F(H, e ; b)=(h+k) b^{r}+\sum_{i=1}^{r-1}\left(2 b^{i}\right)+1$. If $n=2 r$, then letting $v$ be the center vertex of a longest path, we choose $e$ to be the edge incident with $v$ along the path from $v$ to the vertex of degree $k+1$, yielding $F(H, e ; b)=h b^{r+1}+k b^{r}+b^{r}+\sum_{i=1}^{r-1}\left(2 b^{i}\right)+1$. Therefore, we show the lower bound holds. Additionally, when $n=1$ or $n=2$, the result follows from Lemmas 3 and 7 .

Suppose $n \geq 3, b \geq\left(\frac{h+1}{h}\right)(k-h)+1$ and $T \rightsquigarrow(H ; b)$. Let $v_{h}$ and $v_{k}$ denote the vertices of degrees $h+1$ and $k+1$ in $H$, i.e. the centers of the $S_{h}$ and $S_{k}$ we constructed $H$ from.

The tree $H^{\prime}$ formed by removing the leaves of $H$ is a $P_{n}$ with leaves $v_{h}$ and $v_{k}$, and $d_{H}\left(v_{k}\right) \geq d_{H}\left(v_{h}\right) \geq h+1$. By Lemma 6 , there is a subtree $T^{\prime}$ of $T$ such that $T^{\prime} \rightsquigarrow\left(H^{\prime} ; b\right)$, $C_{v}\left(T^{\prime}\right)$ forbids $V\left(H^{\prime}\right)$ for all $v \in V\left(T^{\prime}\right)$, and $\left|L_{u}\left(T^{\prime}\right)\right| \geq h b$ for all $u \in L\left(T^{\prime}\right)$. For notational convenience, we use $d^{\prime}, V^{\prime}$ in place of $d_{T^{\prime}}$ and $V\left(T^{\prime}\right)$, and in what follows we will only consider clumps of $T^{\prime}$.

Consider the following partition of $V^{\prime}$ (some sets may be empty):

$$
\begin{aligned}
L_{k} & =\left\{v \in V^{\prime}: d^{\prime}(v)=1,\left|C_{v}\right| \geq k b\right\}, \\
L_{h} & =\left\{v \in V^{\prime}: d^{\prime}(v)=1,\left|C_{v}\right|<k b\right\}, \\
B & =\left\{v \in V^{\prime}: d^{\prime}(v) \geq b-(k-h)+1\right\}, \\
S_{B} & =\left\{v \in V^{\prime}: 2 \leq d^{\prime}(v) \leq b-(k-h),\left|C_{v}\right| \geq(k-h) b\right\}, \text { and } \\
S_{S} & =\left\{v \in V^{\prime}: 2 \leq d^{\prime}(v) \leq b-(k-h),\left|C_{v}\right|<(k-h) b\right\} .
\end{aligned}
$$


We know that $T^{\prime} \rightsquigarrow\left(P_{n} ; b\right)$, and by Lemma $1 T^{\prime}$ has a large number of vertices of large degree. By Corollary $2, P_{n} \in \mathcal{S}$ and $T^{\prime}$ therefore has at least the "right" number of leaves, in the context of the associated $b$-blow-up. We might further expect $T^{\prime}$ to contain the right number of leaves that can correspond to $v_{k}\left(L_{k}\right)$ and the right number that correspond to $v_{h}\left(L_{h}\right)$ and the bound would immediately follow. This does not necessarily happen, and the $L_{k}$ can fail to provide the bound we need. We will show that this deficiency is balanced out by vertices of large degree in $T^{\prime}$ (implying many leaves of $T^{\prime}$ ), and vertices of small degree but with a large contribution in their clumps $\left(S_{B}\right)$. Vertices of smaller degree that are "too small" $\left(S_{S}\right)$ will be accounted for by the coloring we construct.

In what follows, let $\hat{H}=H\left[V(H) \backslash\left(\left\{v_{h}\right\} \cup L(H)\right)\right]=H^{\prime}-v_{h}$.

Claim 9. Let $v \in L_{h}$. Then $\{v\} \cup C_{v}$ forbids $V(\hat{H})$.

Proof of Claim 9. Let $u \in V^{\prime}$ such that $u v \in E^{\prime}$, and let $W=N(v) \backslash N^{\prime}(v)$. We can greedily partition $W$ into $N_{1}, \ldots, N_{s+1}$, where for $1 \leq i \leq s, N_{i} \ni w_{i}$ so that $\sum_{w \in N_{i}} d(w) \geq$ $b+1$ and $\sum_{w \in N_{i} \backslash\left\{w_{i}\right\}} d(w) \leq b$, and $\sum_{w \in N_{s+1}} d(w) \leq b$. Since $\sum_{w \in W} d(w) \leq\left|C_{v}\right|<k b$, $s \leq k-1$, and if $s=k-1$, then $\sum_{w \in N_{s+1}} d(w) \leq b-k$.

For $1 \leq i \leq s$, color all edges incident with vertices in $N_{i} \backslash\left\{w_{i}\right\}$ with color $i$, and color edge $v w_{i}$ with color $k$. Color all edges incident with vertices in $N_{s+1}$ with color $s+1$. Additionally, since we have used color $k$ at most $(k-1)+(b-k)=b-1$ times, color $u v$ with color $k$.

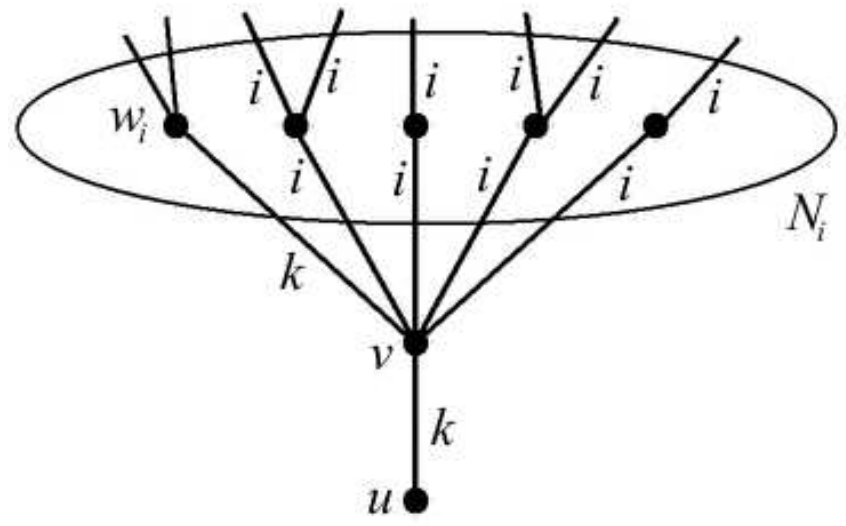

Figure 3: The partial coloring on edges incident with $\{u, v\} \cup N_{i}, 1 \leq i \leq s$.

Since there are at most $k b$ edges incident with $\{v\} \cup C_{v}$, and we have used colors $1,2, \ldots, s+1$ at most $b$ times each, color the remaining uncolored edges (which are incident with $C_{v}$ ) with $1,2, \ldots, k$. Extend this to a coloring of all of $T$, and suppose $v$ lies in a rainbow $H$. If $v$ is not a leaf, then some $x$ in $C_{v}$ serves as a vertex in $H$. Since edges incident with $C_{v}$ are $k$-colored, $x$ cannot correspond to $v_{k}$, so a rainbow path from $x$ to $u$ must exist. Since $u v$ has color $k$, this path must include some $w \in W \backslash\left\{w_{1}, \ldots, w_{s}\right\}$. But $w$ only sees one color, which implies $x=w$, and that $v$ corresponds to either $v_{h}$ or $v_{k}$. As $v$ only sees at most $k$ colors, it must be $v_{h}$, and $\{v\} \cup C_{v}$ forbids $V(\hat{H})$.

Claim 10. Let $v \in S_{S}$. Then $\{v\} \cup C_{v}$ forbids $V(\hat{H})$. 
Proof of Claim 10. Constructing the same sets $W, N_{1}, \ldots, N_{s+1}$ as in the proof of Claim 9 , we see that $s \leq k-h-1$, but instead, our coloring will use $k-h+1$ colors, and color $k-h+1$ is applied to edges $v w_{i}$, and to edges of the form $u v$, where $u \in V^{\prime}$. Since $s \leq k-h-1$ and there are at most $b-(k-h)$ such $u$, color $k-h+1$ is applied at most $b-1$ times. Since $k-h+1 \leq k$, the result follows from same argument as in Claim 9 .

Claim 11. $|B|+\left|S_{B}\right|+\left|L_{k}\right| \geq A R\left(P_{n-1} ; b\right)+1$.

Proof of Claim 11. Let $S_{0}=S_{S} \cup L_{h}$. We know that every $C_{v}, v \in V^{\prime}$, forbids $V\left(H^{\prime}\right)$ and consequently forbids $V(\hat{H})$. By Claims 9 and 10, for all $x \in S_{0},\{x\} \cup C_{x}$ forbids $V(\hat{H})$. $T^{\prime} \rightsquigarrow\left(P_{n} ; b\right)$ with $n \geq 2$ shows that $B \neq \emptyset$ by Lemma 1 , so $S_{0}$ is a proper subset of $V^{\prime}$.

Applying Lemma 5, we therefore have $\left|V^{\prime} \backslash S_{0}\right| \geq A R(\hat{H} ; b)+1$, and the rest follows from noting $\left|V^{\prime} \backslash S_{0}\right|=|B|+\left|S_{B}\right|+\left|L_{k}\right|$ and $\hat{H}$ is a $P_{n-1}$.

We have now established all of the bounds we need for the result: Since

$$
e(T)=e\left(T^{\prime}\right)+\sum_{v \in V^{\prime}}\left|C_{v}\right|
$$

and $e\left(T^{\prime}\right) \geq A R\left(P_{n} ; b\right)$, we bound $\sum_{v \in V^{\prime}}\left|C_{v}\right|$. First, notice

$$
\sum_{v \in V^{\prime}}\left|C_{v}\right| \geq h b\left|L\left(T^{\prime}\right)\right|+(k-h) b\left(\left|L_{k}\right|+\left|S_{B}\right|\right)
$$

By Lemma 1 , letting $X=A R\left(P_{n-2} ; b\right)+1$, since $b+1 \geq b-(k-h)+1$ we have $|B| \geq X$ and

$$
\begin{aligned}
|L(T)| & \geq \sum_{v \in B} d^{\prime}(v)-2(|B|-1) \\
& \geq(b+1) X+(b-(k-h)+1)(|B|-X)-2(|B|-1) \\
& =(b-1) X+(b-(k-h)-1)(|B|-X)+2 \\
& =A_{1}+A_{2}(|B|-X),
\end{aligned}
$$

with $A_{1}=(b-1) X+2$ and $A_{2}=b-(k-h)-1$. Combining this with Claim 11, we have

$$
\begin{aligned}
\sum_{v \in V^{\prime}}\left|C_{v}\right| & \geq h b\left(A_{1}+A_{2}(|B|-X)\right)+(k-h) b\left(1+A R\left(P_{n-1} ; b\right)-X-(|B|-X)\right) \\
& \geq h b A_{1}+(k-h) b\left(1+A R\left(P_{n-1} ; b\right)-X\right)
\end{aligned}
$$

provided $h b A_{2}-(k-h) b \geq 0$. Rewriting, we see that we simply need $b \geq\left(\frac{h+1}{h}\right)(k-h)+1$, the condition posited in the statement of this theorem. 
We now prove the main result in the even case, so assume $n=2 r$. Applying Corollary 2 to our bound on $\sum_{v \in V^{\prime}}\left|C_{v}\right|$, we get

$$
\begin{aligned}
A_{1} & =(b-1) X+2 \\
& =(b-1)\left(A R\left(P_{n-2} ; b\right)+1\right)+2 \\
& \geq(b-1)\left(1+\sum_{i=1}^{r-2} 2 b^{i}+b^{r-1}+1\right)+2 \\
& =b^{r}+b^{r-1},
\end{aligned}
$$

and

$$
\begin{aligned}
1+A R\left(P_{n-1} ; b\right)-X & =A R\left(P_{n-1} ; b\right)-A R\left(P_{n-2} ; b\right) \\
& =\left(1+\sum_{i=1}^{r-1} 2 b^{i}\right)-\left(1+\sum_{i=0}^{r-2} 2 b^{i}+b^{r-1}\right) \\
& =b^{r-1},
\end{aligned}
$$

yielding $\sum_{v \in V^{\prime}}\left|C_{v}\right| \geq h b^{r+1}+k b^{r}$. Combining this with our bound on $e(T)$, we see that

$$
e(T) \geq\left(1+\sum_{i=1}^{r-1} 2 b^{i}+b^{r}\right)+\left(h b^{r+1}+k b^{r}\right) .
$$

The proof for $n$ odd is analogous.

\section{Acknowledgements.}

The author wishes to thank his advisor, Tom Bohman, for the invaluable insights he provided, as well as the anonymous referee, whose comments were of immense value in improving the clarity of the paper. The author would also like to express profound gratitude to L. Charles Biehl, who introduced him to graph theory, and who has been a constant source of encouragement. Thank you, Mr. Biehl.

\section{References}

[1] T. Bohman, A. Frieze, O. Pikhurko and C. Smyth, Anti-Ramsey Properties of Random Graphs, manuscript.

[2] P. Erdős, M. Simonovits and V. T. Sós, Anti-Ramsey Theorems, Colloquia Mathematica Societatis János Bolya 10, Infinite and Finite Sets, Keszethely, 1973.

[3] H. Lefmann, V. Rödl and B. Wysocka, Multicolored Subsets in Colored Hypergraphs, Journal of Combinatorial Theory A 74 (1996) 209-248. 